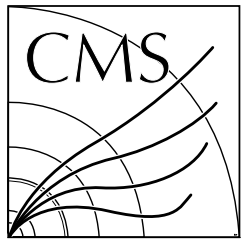

The Compact Muon Solenoid Experiment
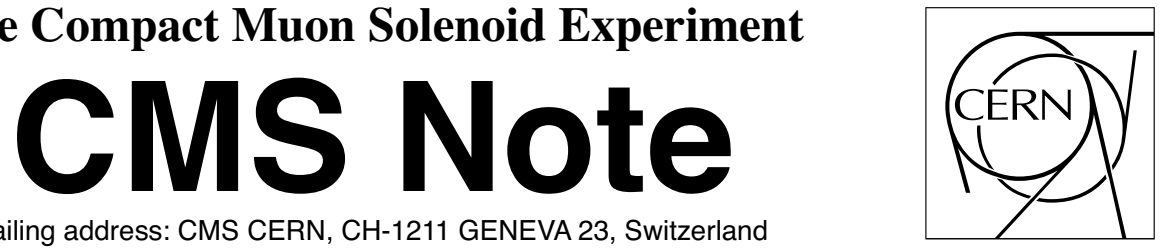

01 April 2008 (v4, 24 November 2008)

\title{
The Detector Control Systems for the CMS Resistive Plate Chamber
}

\author{
P. Paolucci, \\ INFN, Naples, Italy \\ G. Polese ${ }^{\text {a) }}$ \\ Lappeenranta University of Technology, Lappeenranta, Finland
}

\begin{abstract}
The Resistive Plate Chamber system is composed of 912 double-gap chambers equipped with about $10^{4}$ front-end boards. The correct and safe operation of the RPC system requires a sophisticated and complex online Detector Control System, able to monitor and control $2 \cdot 10^{4}$ hardware devices distributed on an area of about $5000 \mathrm{~m}^{2}$. The RPC DCS acquires, monitors and stores about $10^{5}$ parameters coming from the detector, the electronics, the power system, the gas, and cooling systems. The DCS system and the first results, obtained during the 2006 and 2007 CMS cosmic runs, are described in this note.
\end{abstract}

a) also CERN, Geneva, Switzerland 


\section{Introduction}

The CMS Resistive Plate Chambers (RPC) system consists of 912 RPCs at its start-up in middle of 2008. A time resolutions of few nanoseconds and a good spatial resolution $(\sim \mathrm{cm})$, make them an optimal choice for the muon trigger[1]. A continuous control and monitoring of the detector, the trigger and all the ancillary sub-systems (high voltages, low voltages, environmental, gas, and cooling), is required to achieve the operational stability and reliability of such large and complex detector and trigger system. The role of the RPC Detector Control System is to monitor the detector conditions and performance, control and monitor all subsystems related to RPC and their electronics, and store all the information in a dedicated database, called the Condition DB. Therefore the RPC DCS system has to assure the safe and correct operation of the sub-detectors during the entire CMS life time (more than 10 years), detect abnormal and harmful situations and take protective and automatic actions to minimize consequential damages.

The RPC DCS hardware is subdivided in several sub-systems (Fig. 1): High Voltage (HV), Low Voltage (LV), environmental (humidity, temperature and pressure), gas, and cooling systems. The monitoring software has been developed using PVSS II [2], the SCADA system chosen by CERN for the LHC experiments, and the Joint Controls Project (JCOP) framework [3]. The present paper is focused on the design and realization of the software applications for the control and monitoring of the HV/LV systems and of the environmental parameters. Cooling and Gas systems [5] are instead developed centrally by CMS and all information are monitored and shared through the Central DCS using the Data Interchange Protocol (DIP) middleware.

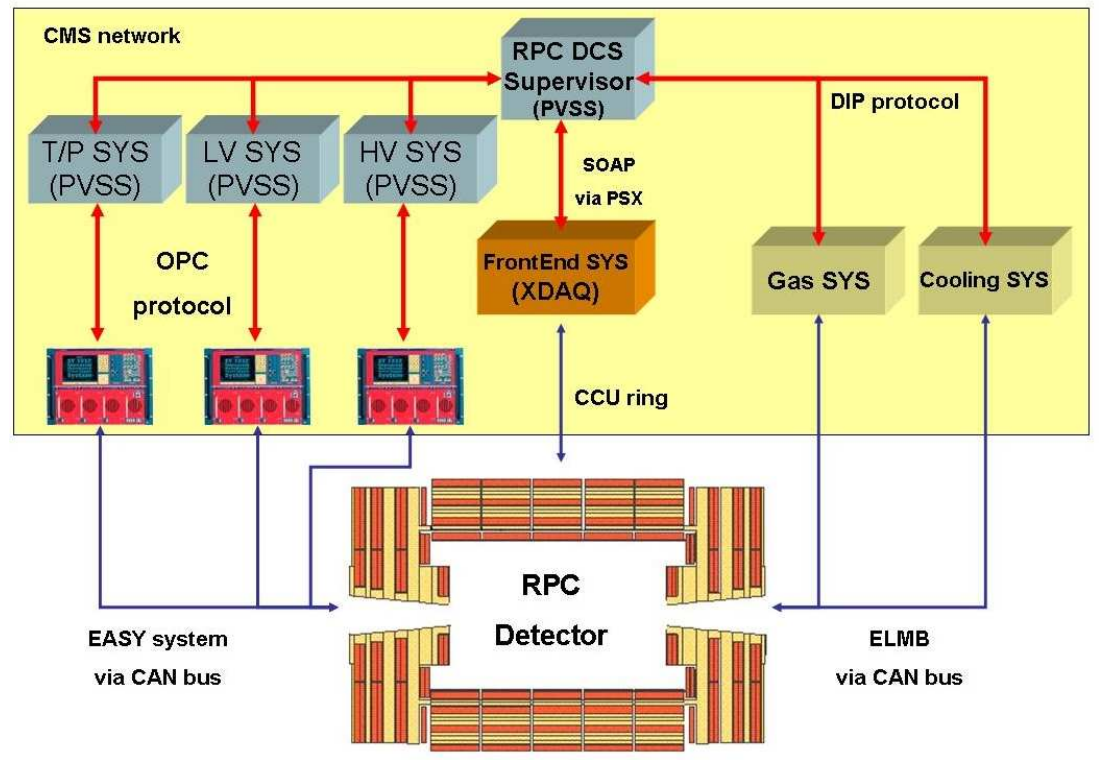

Figure 1: The CMS RPC detector control system layout

\section{Hardware Architecture}

\subsection{RPC system description}

The RPC system is divided in two regions: barrel $(0<|\eta|<1.2)$ and endcap $(0.9<|\eta|<1.6)$. The barrel is made by 5 wheels each with 12 sectors, equipped with 4 muon stations. The two innermost stations are made of a Drift Tube chamber (MB1 or MB2), surrounded by two RPCs (RB1-in, RB1-out and RB2-in, RB2-out). The two outer muon stations are made by a DT chamber (MB3 and MB4) coupled with two RPCs (RB3 and RB4). In some special sectors there are one RB4 (sector 9 and 11) or four RB4 (sector 4). In total there are 4 muon station and 6 RPC layers used for triggering and detect muons. Each endcap is made by 3 iron disks and 4 muon stations of Cathode Strip Chamber and RPC. The muon station contains one layer of double-gap RPC and is divided in R into 3 chambers (REs/1, REs/2 and REs/3, where $s=1, \ldots, 4$ is the station number). Chambers RE2/1, RE3/1 and RE4/1 cover $20^{\circ}$ in $\phi$, whereas all the other RE cover $10^{\circ}$. 


\subsection{RPC chamber description}

An RPC gap is made of two parallel bakelite plates $\left(1-210^{10} \Omega \mathrm{cm}\right)$ placed at a distance of $2 \mathrm{~mm}$ and filled with a gas mixture of $94.7 \% \mathrm{C}_{2} \mathrm{H}_{2} \mathrm{~F}_{4}, 5 \% \mathrm{i}-\mathrm{C}_{4} \mathrm{H}_{10}$ and $0.3 \%$ of $S F_{6}$. High voltage is applied to the outer graphite coated surface of the bakelite plates in order to have an electric field inside the gas gap able to generate a charge avalanche along the track of an ionizing particle. The avalanche induces a signal on the aluminum strips placed outside the gap and isolated from the graphite. A barrel RPC chamber schema, with two double-gaps and a strip plane in the middle, is shown in Figure 2. There are 16 different typologies of chambers but all of them are made by two or three double gaps assembled in a common mechanical framework, with a read-out strips plane placed in between. Chambers are equipped with two or three rows of 6 front-end boards [4] each connected to 16 readout strips. The total number of FEBs per chamber is always 12 or 18 (three double gaps) and 10 in some special smaller RB4. The high voltage connection of the 2 or 3 upper and lower gaps are joined together (Fig. 2) in order to reduce the number of $\mathrm{HV}$ channels keeping the possibility to have different gap voltages in every chamber.

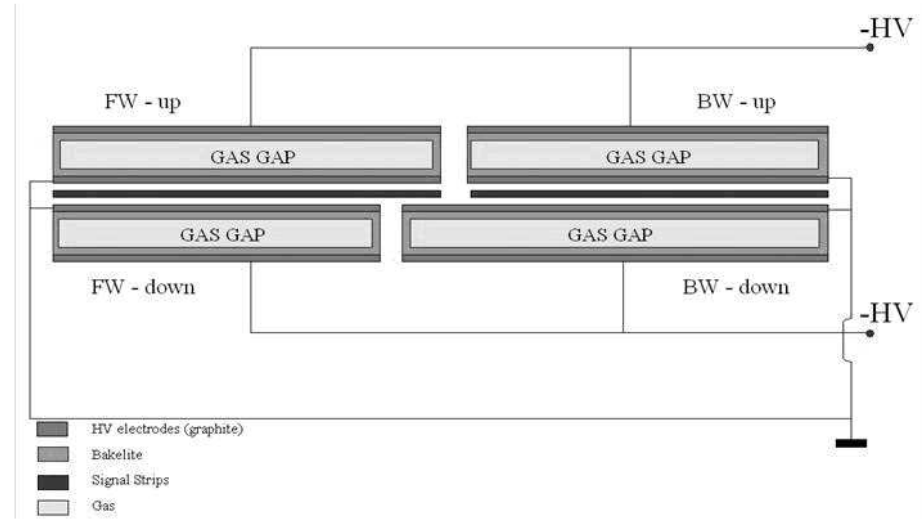

Figure 2: A barrel RPC chamber made by two double-gaps and with a strip plane in the middle.

\subsection{The Power system : high voltage and low voltage}

The complexity and high granularity of the RPC system and the hostile environment (high magnetic field and high radiation flux) in which it will operate, imposed challenging constraints in the development of the power distribution system. In the muon system, a large part of the power system is located close to the detector and in particular inside the racks placed on the balconies around the barrel wheel and the endcap disk. In this area the magnetic field can reach up to $6 \cdot 10^{-2}$ Tesla, while the radiation is up to $10^{7}$ proton $/ \mathrm{cm}^{2}$ and $5 \cdot 10^{10}$ neutron $/ \mathrm{cm}^{2}$. The power system has been designed taking into account the environmental requirements and the necessity to minimize the probability to have dead or inefficient regions due to the failure of some power supply channels. Every RPC chamber has been equipped with two independent HV channels (one per layer) and two LV channels choosing a good compromise between the cost and granularity. In addition four low voltage channels are needed to supply each Link Boards Box (LBB), aimed to collect data from each chamber, synchronize and send them to trigger and readout chain in control room.

In conclusion the entire RPC power system consists of :

- 912 high voltage channels,

- $\simeq 1000$ low voltage channels for front end boards on the chambers,

- $\simeq 300$ low voltage channels for the link boards.

The solution chosen by the RPC collaboration for the power system is based on the CAEN EASY [7](Embedded Assembly SYstem) project, which consists of components made of radiation and magnetic field tolerant electronics and based on a master-slave architecture. Branch controller boards, located inside the CAEN SY1527 mainframe, act as master, and are aimed to control and monitor one or more slaves. The masted is placed in a safe and accessible area as the electronic room. The slave is where the power is generated and is designed to be modular and multifunctional in order to be able to have mixed systems containing both HV and LV power supplies. It 
is based on a crate with a dedicated backplane, housing a certain number of different boards: power supplies, $\mathrm{ADC}$, and DAC. The slave system can be placed close to the detector, in a hostile and not accessible area and for this reason is modular, redundant and based on radiation tolerant electronics. Nevertheless, the RPC collaboration decided to collocate the entire HV system in an accessible area (counting room) to easily fix any problem regarding the connection and the distribution of the HV. The LV system, instead, has the slave part (power supply) placed in racks surrounding the detector and the master in counting room.

Table 1: Requirements for the HV and LV system for RPC Chambers

\begin{tabular}{|c|c|c|c|}
\hline Power Supply & High Voltage & LowVoltage FEB & Low Voltage for LBB \\
\hline Hostile Environment & Yes & Yes & Yes \\
\hline Voltage & $12 \mathrm{kV}$ & $7 \mathrm{~V}$ & $4 \mathrm{~V}$ \\
\hline Current & $1 \mathrm{~mA}$ & $3 \mathrm{~A}$ & $14 \mathrm{~A}$ \\
\hline Ripple & $<100 \mathrm{mV} \mathrm{pp}$ at load & $<10 \mathrm{mV}$ pp at load & $<10 \mathrm{mV}$ pp at load \\
\hline Programmable Voltage & $0-12 \mathrm{kV}$ & $0-9 \mathrm{~V}$ & $0-5 \mathrm{~V}$ \\
\hline Monitored Current Precision & $0.1 \mathrm{uA}$ & $100 \mathrm{~mA}$ & $100 \mathrm{~mA}$ \\
\hline Monitored Voltage Precision & $<10 \mathrm{~V}$ & $100 \mathrm{mV}$ & $100 \mathrm{mV}$ \\
\hline Trip Settings & $0-100 \mathrm{~s}$ & $0-10 \mathrm{~s}$ & $0-10 \mathrm{~s}$ \\
\hline
\end{tabular}

\subsection{Environmental Sensor network}

The performance of the RPC detector are strongly related to the temperature and humidity. In particular the noise rate and the dark current of the chamber depend on these two parameters. For this reason monitoring the gas temperature, the humidity and the temperature outside of the chamber is crucial. The environmental sensor network is composed by 550 sensors to measure the temperature of the iron gaps, where the chambers are placed, 40 sensors to measure the temperature of the gas and 40 relative humidity sensors. The temperature sensor is the AD592BN, made by Analog devices, whereas the sensor HIH4000 is used to measure the relative humidity. They assure the robustness, reliability and precision required and can operate in the radiation and magnetic field environment as described in Table 2. Both types of sensors are powered and read by the CAEN ADC (A3801A) boards, equipped with 128 channels and a $12 \mathrm{~V}$ input stage. All ADC boards are placed in the balcony around the detector, in the same EASY3000 crates used for LV.

Table 2: Requirements for the environmental network for RPC Chambers

\begin{tabular}{|c|c|c|}
\hline Environmental sensor & Temperature & Humidity \\
\hline Hostile Environment & Yes & Yes \\
\hline Input range & $-10^{\circ} \mathrm{C}+60{ }^{\circ} \mathrm{C}$ & $0-100 \% \mathrm{RH}$ \\
\hline Accuracy on Measurement & $0.1^{\circ} \mathrm{C}$ & $\pm 2.5 \% \mathrm{RH}$ \\
\hline
\end{tabular}

\section{RPC DCS software}

In accordance with the CMS official guidelines [6], all RPC DCS applications have been developed using the commercial ETM SCADA (Supervisory Control And Data Acquisition) software, PVSS 3.6 [2] and the standard Joint Control Project (JCOP) framework components [3]. Due to the large amount of devices from different subsystems (HV, LV, environment, gas, and cooling), the control and monitoring has to be done in parallel and distributed over different machines. To implement this kind of structure, the DCS uses the finite state machine (FSM) approach and its granularity follows the RPC subsystem structure (wheel, sector, chamber,...). All the subsystems are handled and controlled by the RPC Supervisor, aimed to gather and summarize all the information and to present a simplified but coherent and general view to the users.

RPC DCS uses most of the functionalities provided by the JCOP+PVSS software as the final state machine, the graphical user interface, the alarm handler and the ORACLE database interface, that allows the storage of the data in the CMS online database and the loading of the hardware configuration from the CMS configuration database.

\subsection{Architecture}

The RPC DCS software architecture has been developed following a hierarchical double-tree structure: geographical and hardware oriented tree (Fig. 3). Both trees give useful information on the system from different points of view. The hardware tree shows the status of all the equipment involved in the operation and allows to find out and 


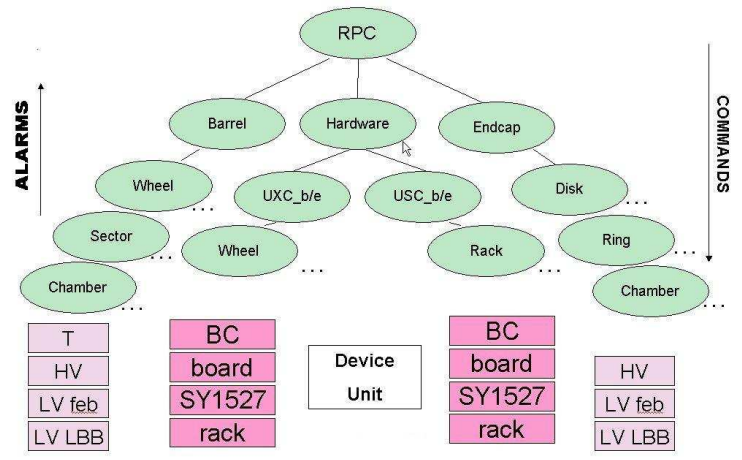

Figure 3: Structure of the hierarchy tree of the RPC DCS. Different branches describe the RPC system from geographical and hardware points of view. All commands go down the hierarchy, while information and error messages are reported upwards.

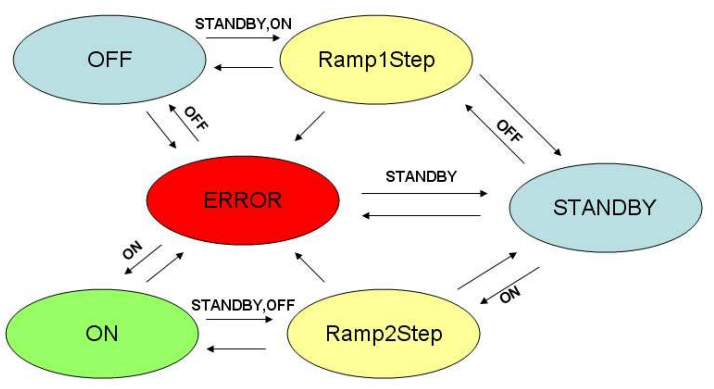

Figure 4: Example of the Chamber Finite State Machine.

handle problems occurring in a particular hardware component, involving several detector parts. The geographical tree, instead, describes the system from the detector point of view, focusing on the location of the each component on the detector. This subdivision closely follows the geometry of the detector, i.e. wheels and sectors for the barrel, discs and rings for the endcap, allowing a close correlation with readout data. PVSS and JCOP framework allow an easy implementation of such a structure. Every tree node is described by predefined objects, Control Unit (CU) and Logical Units (LU) that, with different task and privileges, are aimed to drive the behavior of the hardware equipments and subsystems under them. In fact they can configure, monitor, and control all child nodes and recover from error state. This facility assures the partitionability and scalability, allowing a robust and powerful management of the system. At the lowest level, as leaves of the tree, there are different logical groups describing the hardware devices: HV, front-end LV, trigger LV power supply, and environmental systems. These tree nodes, representing electronic channels, are described instead by Device Units (DUs). Each DU is the interface to the hardware component; it translates the received commands, understands the device states and generates eventual alarms. The HV and LV channels, the power supplies, and the slave crates are managed separately through dedicated DUs. The root (top) node of the RPC DCS is connected directly to the CMS central DCS system and is used to communicate and exchange actions, states and commands. The commands, coming from the central DCS, are propagated through the RPC FSM tree down to the devices. Here they are interpreted accordingly as hardware commands. The hierarchical tree structure allows only vertical data flow: commands move downwards, while alarms and state changes propagate upwards (Fig. 3).

\subsection{The Finite State Machine}

In order to fulfil a high rate of automation in control processes, reduces human errors, unavoidable in repetitive action, and optimizes recovery procedures in case of undesired states, all the RPC DCS hierarchy nodes are implemented through a Final State Machine (FSM) mechanism. It offers an easy, powerful and safe way to get the full detector control. In fact it allows to summarize the detector status through a limited number of states, drive it to predefined configurations and translate all the operation mode in simple actions, hiding to the operator the complexity of the actions required. The FMS toolkit in PVSS is based on SMI++ [8] and provided by the JCOP framework. The states and the commands for the top nodes as well as the conjunction nodes have been chosen by CMS in order to have a uniform structure. The states are: ON, OFF, STANDBY, and ERROR and the commands are: ON, OFF, and STANDBY. The use of these particular states and commands ensures uniformity and compatibility with the central CMS DCS, permitting adequate transitions between the states. Their small number and general definition makes them suitable for all sub-detectors. The states from central DCS are translated in meaningful states for RPC. For this reason a transitional state (RAMPING) has been added to the previous state. It describes the situation in which the high voltage of one or more chambers is ramping up or down. The STANDBY state is used for the RPC detector as a safe state in which the LV channels are ON, while the high voltages are at an intermediate and safe value. This state has been implemented for test and calibration runs or for period with a 
"not stable" beam and magnet ramping conditions.

\subsection{The Graphical User Interface (GUI)}

The GUI is developed to be an intuitive tool to control and monitor the detector, easy to use also by non-experts and able to preserve the system from any dangerous action. It is a collection of panels in PVSS language and offers the following functionalities:

- an easy navigation throughout the entire system structure, thanks to a combination of text, graphical objects and synoptic diagrams;

- visualization and setting of any process variable;

- global parameter setting, thus speeding operations and reducing human errors;

- plots, diagrams, histograms, and table for a quick visualization of the evolution of a parameter in time;

- complete visualization of the alarm condition on all critical elements.

To fulfill the above functionalities, approximately 40 panels (tree structure) have been designed following the naming conventions and the color codes decided centrally by the CMS DCS group. Examples of RPC DCS panels are shown in Figure 5 and 6. The GUI allows a complete control of the entire RPC system and therefore, to prevent any human error, a different access levels have been set. The following self-explanatory groups: PVSS expert, RPC expert, and RPC user have been successfully tested during our pilot runs. An indispensable feature of any control system is the alarm handling. An alarm is issued every time the system unwontedly leaves the desired state or if a given parameter deviates from a predetermined range. A sets of alarm conditions, severity levels, and recovery procedures have been defined and implemented for all the critical hardware parameters in order to have very fast information regarding any abnormal condition. The presence of an alarm is promptly signaled, combined with the information about the origin and the severity level, by the PVSS alarm handling system.

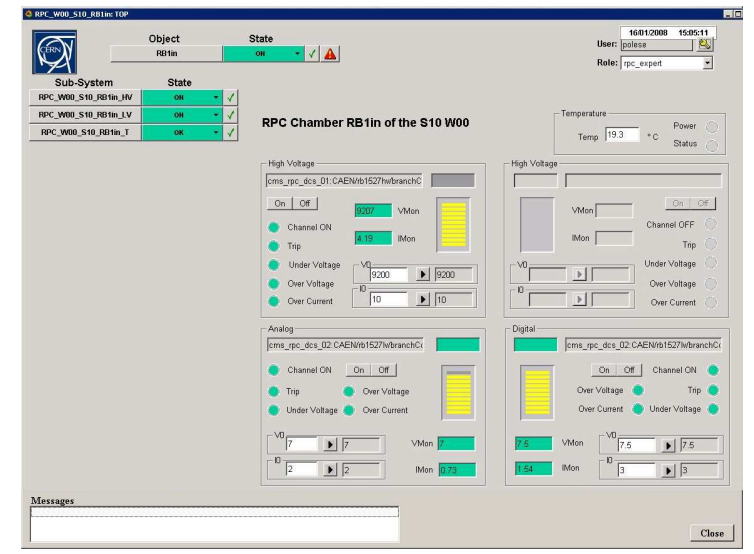

Figure 5: Example of the panel describing the chamber parameters with a detailed overview of all chamber working parameters and alarm conditions.

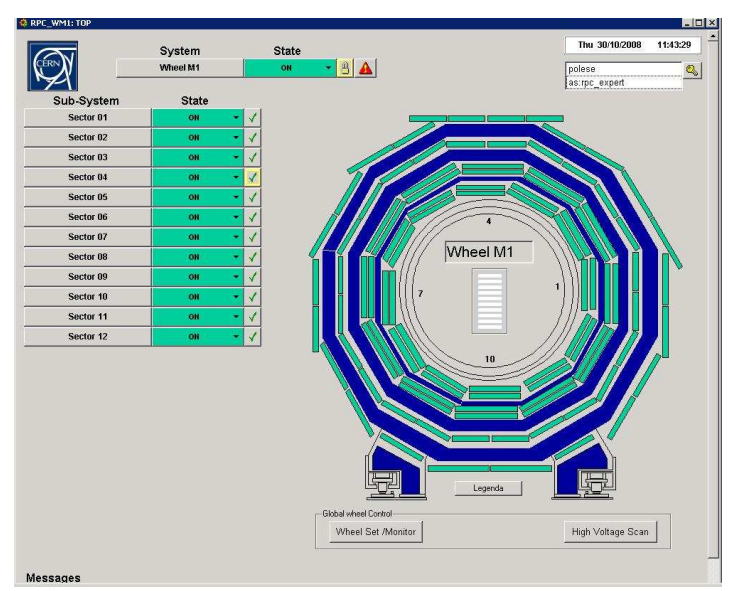

Figure 6: Layout of the Barrel Wheel panel. It offers an overview of the children nodes status (on the left), a visual summary of the status of all selected wheel chambers and the possibility to set and monitor all working parameters, depending on the user privileges.

\subsection{Integration in central DCS and Run Control}

The RPC DCS is directly connected with the central DCS. In this way the central DCS propagates commands, alarm and messages directly to the RPC DCS and publish to the CMS Run Control the RPC status condition 
during the data taking by means of the FSM (Fig. 7). In this way a bidirectional communication between the RPC DCS and run control is provided, in order to synchronize the status of the detector with the physics data taking operation. The RPC DCS is also able to operate in standalone mode, in order to be used during the commissioning and calibration phase, by means of a direct connection to the RPC Run control. This allows us to synchronize the configuration operations among different RPC parts, check the status of the entire RPC system and manage centrally the warning and error messages coming from different RPC partitions.

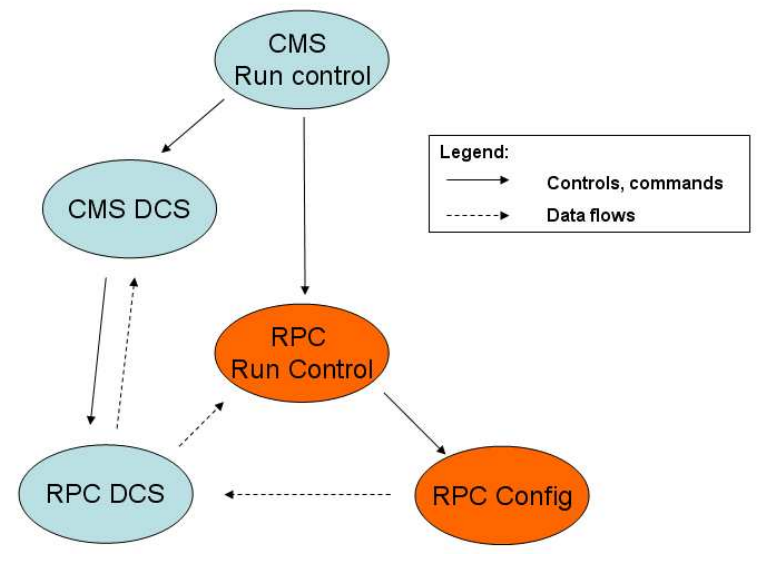

Figure 7: Logical layout RPC DCS with other DAQ subsystems

\subsection{Database}

The hardware description and the configuration of the RPC system is particularly laborious, due to the large number and heterogeneity of the elements, and require data base infrastructures to keep track of the different running configurations. All the structural information, geographical position and configuration parameters, necessary to put the detector in running condition, are stored in a central relational database, called Configuration database, that is based on the ORACLE technology.

On the other hands, all the information regarding the running conditions and non-event data controlled by RPC DCS need to be stored in order to monitor the system behavior over time. For this purpose a Conditions database, based on ORACLE technology as well, is used to archive all parameters relevant to both electronics and detectors performances. These information allow the optimization of the working condition and studies of the equipment response during different phases of the experiment and are a powerful tool to understand the ageing behavior of the detector. In the final configuration the amount of RPC DCS data stored would be about 30 GByte per year, almost $1 / 500$ of the monitored information. The biggest part of these data is represented by chamber dark current. The interface between PVSS and the different databases is developed using a dedicated PVSS manager for ORACLE DBs, able to assure reliability, redundancy, and stability of the storage system.

\section{The RPC DCS commissioning}

The RPC DCS has been extensively tested during the CMS global runs took from July to September 2006 and from September to December 2007. During the global runs only a fraction of the RPC hardware system (about 10\%) was used and an ad-hoc DCS software system was developed and tested.

The RPC chambers were operated with the final configuration of the power systems, CMS DAQ software, data quality monitor (DQM), and DCS were implemented for the detector readout and control. The DCS proved to be a reliable tool for the safe and correct operation of the detectors and trained shifter, were able to operate the detector in a easy and safe way. The RPC DCS has been also successfully integrated into the Central DCS and was able to publish its state and receive command from it. After a short debug phase, the system ran without problems for the entire test period. It was proved to be able to manage properly the interruptions occurred, due to power failures and communication problem with the power supply. The developed FSM adequately followed the detector's behavior and never lost control of the hardware.

The software applications were split on three servers to share load in a configuration similar to the final one (5 servers). Although the hardware used was only the $10 \%$ of the final system, the global runs were an important opportunity to figure out the hardware and software performance using a system as close as possible to the final 
one. One of the most crucial question marks was the hardware communication, the stability of the OPC server and its reliability using different clients with a large number of hardware channels (software components). The OPC server used was the "CAEN OPC server", version 2.12. On the client side, the OPC was configured to manage more than 1000 items per PC with a fixed refresh rate of $2 \mathrm{~s}$. This solution was proven to be optimal during the global runs, but not representative since the limited number of hardware elements used (up to $10 \%$ of the final configuration). Preliminary studies on timing performance with an increasing number of hardware channels was performed in Prevessin CERN site using this OPC server/client configuration and showed a reasonable and effective behavior in the switching and setting operations ( Fig. 9).

Regarding databases, an ad-hoc configuration version describing $80 \%$ of the barrel hardware was stored in the CMS Configuration database. Connection and communication was proven to be stable, reliable and satisfactory. About 1 GByte of DCS data were collected and stored in the CMS condition database during the data taking periods and are now used in the offline analysis of the detector and trigger performance (Fig. 8).

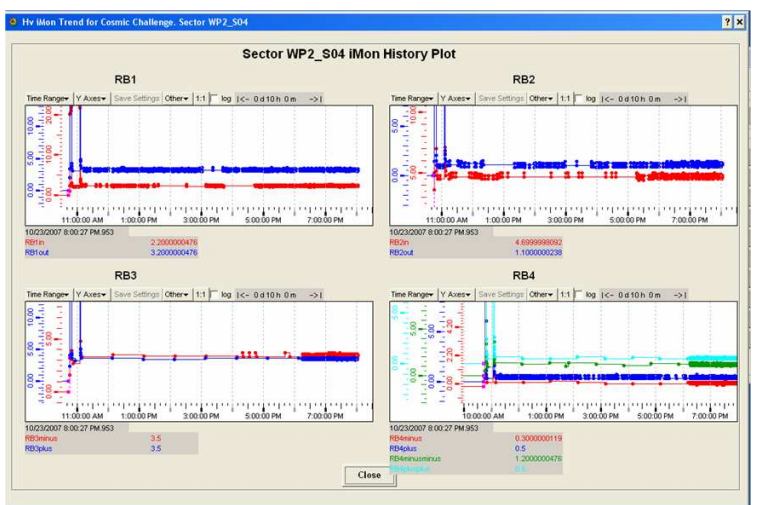

Figure 8: Dark chamber current monitoring of one sector (2007 Global Run)

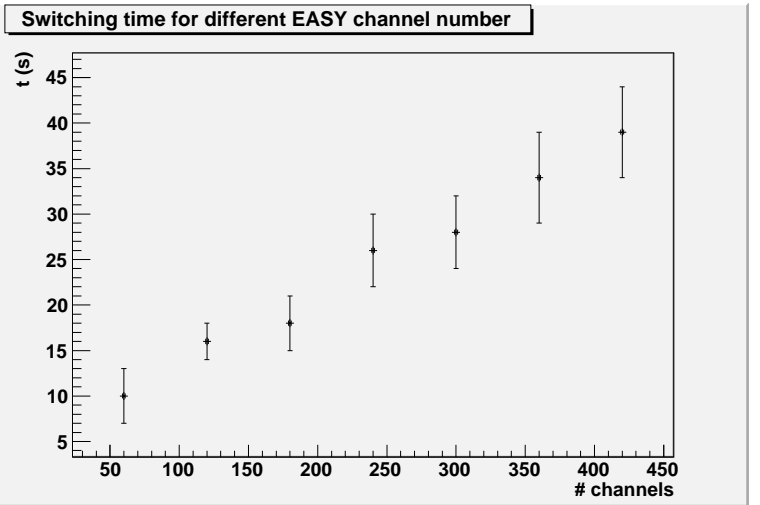

Figure 9: Time required to switch on different LV channels using PVSS and CAEN OPC Server, version 2.12

\section{Conclusions}

The design and development of the RPC detector control system is now almost finished and we are currently working on the installation and commissioning of both hardware and software components. Almost $80 \%$ of the barrel HV and LV system have been delivered and tested intensively at CERN and the installation of the hardware in the CMS underground cavern is now in progress. The entire environmental sensor network has been put in place and all connections have been tested.

The good results obtained during the 2006 and 2007 global runs and the tests performed at CERN with about 400 channels, corresponding to $20 \%$ of the entire system, demonstrated that the RPC DCS was well designed and was able to run in a very stable and safe way for long period.

In conclusion, the RPC DCS system, developed following the guidelines of the DCS central group was proved to be a reliable tool and it is ready to become fully operationally for the summer 2008 when CMS will begin to take data.

\section{References}

[1] CMS Collaboration, "Muon Project”, CERN/LHCC 94-38, 15 December 1997.

[2] "ETM professional control: PVSS (Prozess Visualisierungs und Steuerungs System) SCADA tool", http://itcobe.web.cern.ch/itcobe/Services/Pvss/welcome.html .

[3] M. Beharrell et al. , “ Technology Integration in the LHC experiments Joint COntrols Project, CHEP01, Beijing, September 2001. http://cern.ch/itco/Projects-Services/JCOP/ . 
[4] M. Abbrescia et al. , "New developments on front end electronics for the CMS Resistive Plate Chambers," Nucl. Instrum. Meth. A 456 (2000) 143.

[5] R. Barillere et al., "LHC GCS: A Homogeneous Approach for the Control of the LHC Experiments' Gas Systems", Proceedings of ICALEPCS 2003, Gyeongju, Korea, 2003.

[6] R. Arcidiacono et al. , "CMS DCS design concepts", prepared for 10th International Conference on Accelerator and Large Experimental Physics Control Systems (ICALEPCS 2005), Geneva, Switzerland, 10-15 Oct. 2005.

[7] CAEN S.p.A. Costruzioni Apparecchiature Elettroniche Nucleari, http://www.caen.it/nuclear/easy info.html .

[8] B. Franek and C. Gaspar, "SMI++: Object oriented framework for designing and implementing distributed control systems", presented at 2004 IEEE Nuclear Science Symposium and Medical Imaging Conference (NSS / MIC), Rome, Italy, 16-22 Oct. 2004. 